\title{
The LHCb Upgrade
}

\author{
Marcel Merk ${ }^{* \dagger}$ \\ Nikhef and Vrije Universiteit \\ E-mail: marcel.merk@nikhef.nl
}

The LHCb detector is designed to search for manifestations of new physics via precision measurements in the heavy flavour sector. Results obtained with the 2010 LHC run demonstrate efficient triggering and a clean selection of beauty and charm signal decays, suppressing backgrounds in the presence of multiple collision pile-up. After having collected data corresponding to an integrated luminosity of $5 \mathrm{fb}^{-1}$, LHCb proposes to upgrade the experiment to acquire 50 $\mathrm{pb}^{-1}$ using a full software trigger at a detector readout frequency of $40 \mathrm{MHz}$. The new trigger scheme requires changes in the front end electronics and the replacement of several subdetectors. As a consequence the physics scope of the experiment can be widened beyond the high precision heavy flavour program to include lepton flavour physics, electroweak physics, QCD and direct searches for new, exotic particles.

The 13th International Conference on B-Physics at Hadron Machines - Beauty2011, April 04-08, 2011 Amsterdam, The Netherlands

\footnotetext{
* Speaker.

On behalf of the LHCb Collaboration.
} 


\section{The LHCb Experiment}

The LHCb detector has been collecting data at $7 \mathrm{TeV}$ collision energy since March 2010. Fig. 1 shows a reconstructed event and summarizes the reconstruction performance for high multiplicity events. Although the LHC had not yet reached the nominal number of bunches in the machine, the $\mathrm{LHCb}$ experiment was already operating at its design luminosity at the end of the run. The performance plots illustrate that the number of interactions per bunch-bunch collision is not limiting the physics program of LHCb. At the end of the run data were collected with $\mu=2$. Experience with the first year of LHC data taking also confirmed that the observed efficiencies for detector, trigger, and event selection efficiencies are in agreement with predicted Monte Carlo values.

After collecting an integrated luminosity of approximately $5 \mathrm{fb}^{-1}$, LHCb proposes to upgrade the detector and modify the trigger scheme to collect approximately $50 \mathrm{fb}^{-1}$ of data. A flexible software-based trigger will allow, in addition to a higher event rate, an extended physics program that includes lepton flavour physics, electroweak physics and exotic searches in addition to heavy flavour physics. As such the LHCb upgraded detector becomes a general purpose experiment in the forward direction. Its detector modifications and physics program are described in a letter-of-intent [1] and summarized below. The measurement sensitivities for the upgraded detector are estimated using the MC simulations validated by the data taken with the current detector.
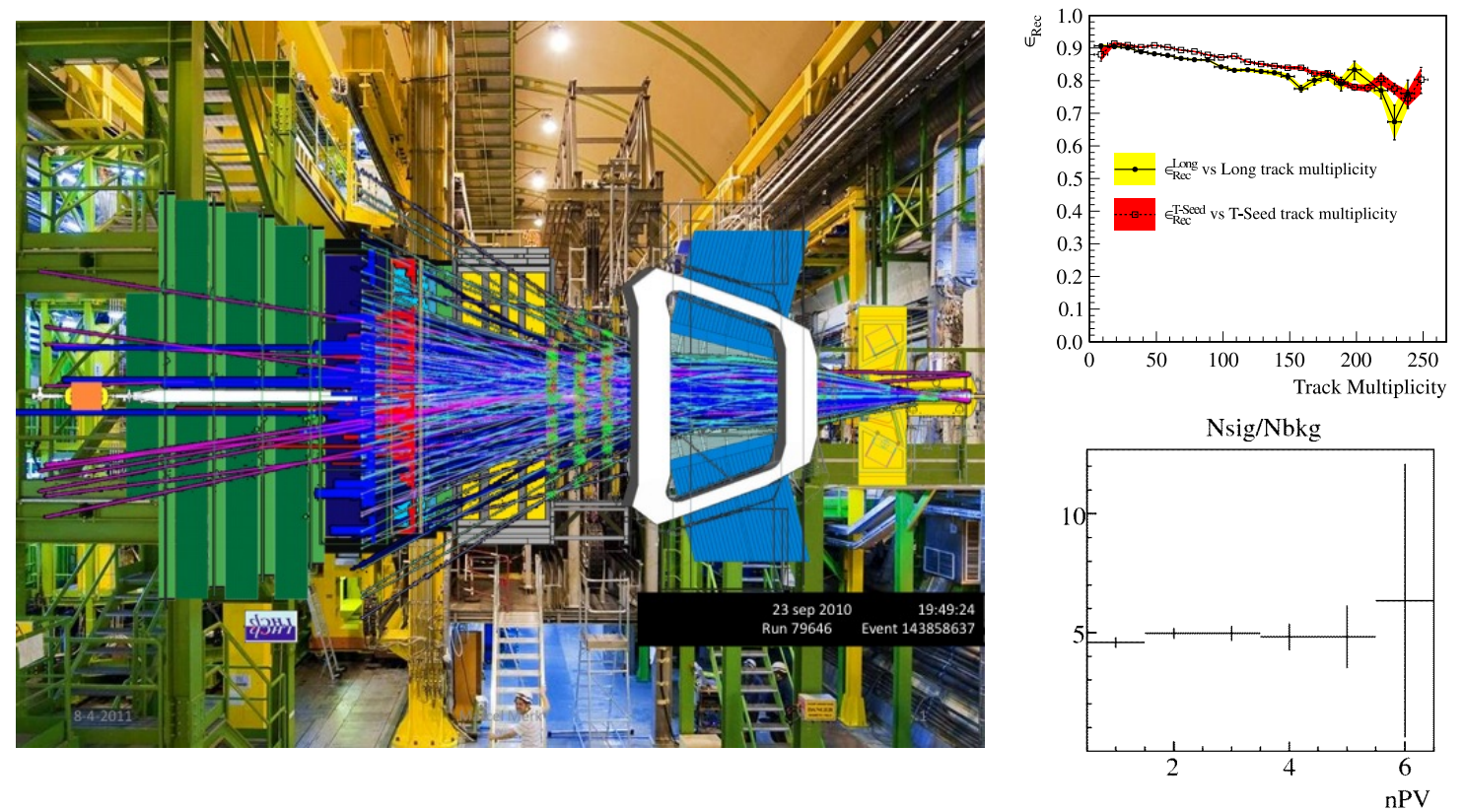

Figure 1: Left: A reconstructed LHCb event together with a schematic detector layout is displayed on top of a photograph of the detector in the experimental hall. Right top: The track reconstruction efficiency for charged particle tracks traversing the full spectrometer ("Long" tracks) as well as tracks in the downstream detector side ("T-Seed" tracks) vs their multiplicity. Right bottom: The signal-to-background ratio within the invariant mass distribution of $J / \psi\left(\mu^{+} \mu^{-}\right) K^{ \pm}$as a function of the number of primary vertices reconstructed in the event. 

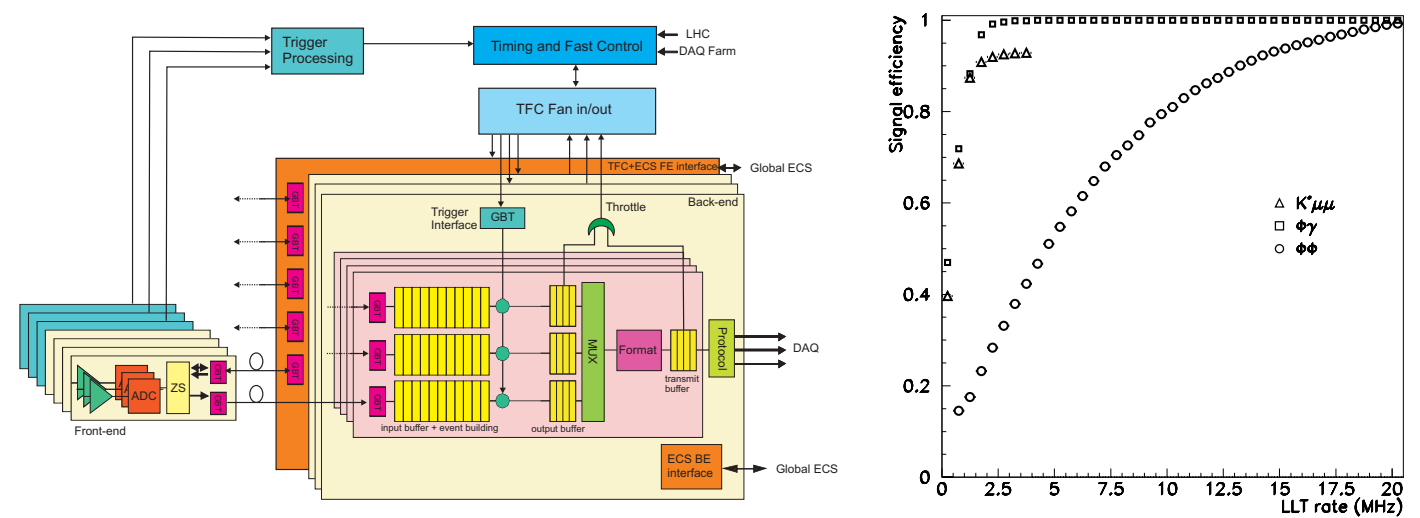

Figure 2: Left: Overview of the general electronics architecture of the LHCb upgrade. Right: The upgraded LLT efficiency for $B_{s} \rightarrow \phi \phi, B^{0} \rightarrow \mu \mu K^{*}$ and $B_{s} \rightarrow \phi \gamma$ decays as function of a possible LLT trigger rate at a luminosity of $10^{33} \mathrm{~cm}^{-2} \mathrm{~s}^{-1}$.

\section{Detector Upgrade}

The LHCb detector was designed for operation in the LHC anticipating nominal instantaneous luminosities between $2-3 \times 10^{32} \mathrm{~cm}^{-2} \mathrm{~s}^{-1}$. The upgraded experiment proposes to increase the luminosity to $1-2 \times 10^{33} \mathrm{~cm}^{-2} \mathrm{~s}^{-1}$, in combination with a full software trigger using a higher detector readout speed. To implement this upgrade, the front end electronics of the sub-detectors are replaced, part of the tracking detectors in the hottest regions are replaced, and a new detector is added for low momentum particle identification. Since the data from all detectors are available in the software trigger, the dedicated level-0 (L0) pre-shower, scintillating pad and first muon station detectors are omitted from the setup.

\subsection{Trigger and Electronics}

The current LHCb detector[2] uses a L0 hardware trigger that selects events with high transverse energy in the calorimeters or high transverse momentum in the muon detector, in order to reduce the event rate to $1 \mathrm{MHz}$. The High Level Trigger (HLT) reconstructs inclusive as well as exclusive B decay topologies and further reduces the event rate to $2 \mathrm{kHz}$ to mass storage. Although the current muon trigger could tolerate a higher luminosity, the calorimeter triggers are saturated and an improvement can only be obtained by increasing the off-detector bandwidth. In the upgrade, the L0 trigger is replaced by a similarly operating Low Level Trigger (LLT) that can be operated in a flexible way to throttle the output rate in the range of 1 to $40 \mathrm{MHz}$. The electronics architecture and the performance of the LLT, at high luminosity running, are shown in Fig. 2. To accomodate an increased readout speed, the Front-End electronics on the detector compresses the digitized signals and transmits them up to $40 \mathrm{MHz}$ over high-speed optical links to the Back-End electronics in the counting house. Details on the readout architecture can be found in [1].

\subsection{Tracking}

The LHCb tracking system consists of a Vertex Locator (Velo) detector surrounding the interaction point and a momentum tracker upstream and downstream of a warm dipole magnet. The detectors upstream of the magnet, the Velo and Trigger Tracker, both use silicon microstrip technology, while downstream of the magnet the main outer region is covered by Outer Tracker drift tubes and the high-occupancy inner region by Inner Tracker silicon strips. 


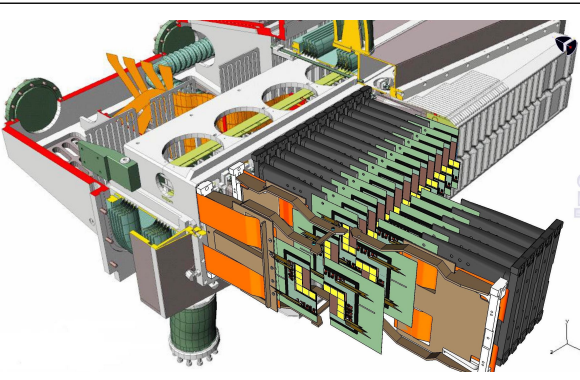

(a)
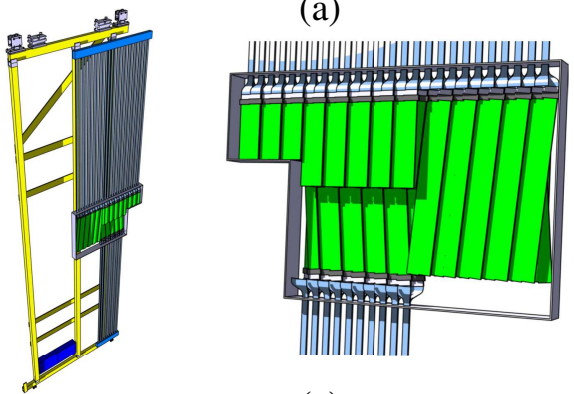

(c)

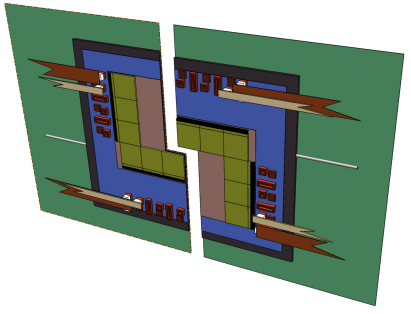

(b)

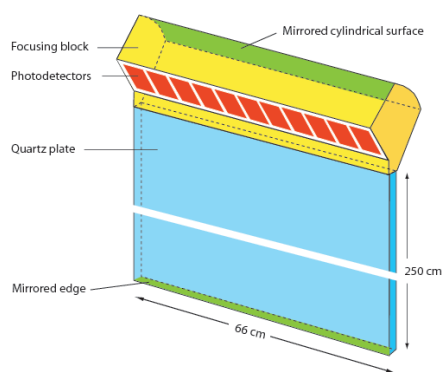

(d)

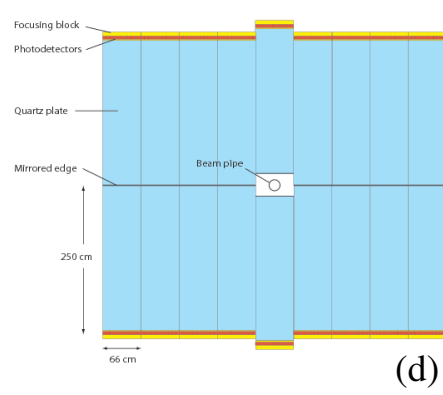

Figure 3: Schematic views of (a): the upgraded Velo detector, (b): a Velo station with Timepix modules, (c): scintillating fiber inner tracker, (d): The TORCH particle identification detector.

The change to a $40 \mathrm{MHz}$ readout scheme necessitates replacement of the silicon strip detectors as the readout electronics is integrated on the detector modules. For the straw tube detectors the electronics can be replaced and the detector modules can be re-used for the upgraded detector.

To accomodate a $40 \mathrm{MHz}$ reconstruction in the Velo the baseline solution foresees a square $55 \mu \mathrm{m}$ granularity $\mathrm{Si}$ pixel detector with a readout solution based on the TIMEPIX chip; named VELOPIX. An alternative option is a radiation hard Si strip detector. An R\&D program investigating optimal hit resolution, minimal radiation thickness $X_{0}$ and efficient cooling is ongoing.

To limit the hit occupancy, several OT detector modules can be replaced by modules with shorter straws. For the Inner Tracker two detector solutions are considered: a new Si strip detector similar to the current IT or, alternatively, a fiber tracker using $0.25 \mathrm{~mm}$ scintillating fibers. Schematic views of the upgrade tracking detectors are shown in Fig. 3(a-c).

\subsection{Particle ID}

Electrons, photons, and muons are identified by the Calorimeter and Muon detectors, while two RICH detectors provide pion, kaon and proton separation in the momentum range from 2 $100 \mathrm{GeV} / \mathrm{c}$. In the upgrade, the RICH system is augmented by a novel time-of-flight detector system to identify low momentum particles, named the TORCH. The detector is schematically depicted in Fig. 3(d). It combines time-of-flight and RICH techniques in $1 \mathrm{~cm}$-thick quartz planes, in which photons propagate in a manner similar to the DIRC detector [3]. The scintillating pad and preshower detectors are no longer needed in the upgrade, while the ECAL and HCAL calorimeter modules can be retained using a reduced HV setting of the photomultipliers. The Muon detector is read out at $40 \mathrm{MHz}$ in the current detector. Its radiation tolerance has been tested at CERN[4].

\section{Physics Program}

The main physics goal of the upgraded detector is to improve the sensitivity for studies of CP 
violation and rare decays in beauty and charm physics. The new trigger scheme, however, allows an extension of the physics program beyond flavour physics to include a wide range of new topics.

\subsection{Quark Flavour Physics}

LHCb is optimized for the study of the physics of quark flavour, with an emphasis on CP violation measurements and rare decays in the $B$-meson system. The measurement sensitivity of the upgraded $\mathrm{LHCb}$ detector for several key channels has been evaluated using simulations. An overview of these sensitivities is given in Tab. 1.

The improved sensitivity for the $\mathrm{CP}$ violation program can be summarized as follows. First, where the current detector expects to find or rule out large deviations from the Standard Model $(\mathrm{SM})$ in the measurement of mixing-induced $\mathrm{CP}$ violation in the $B_{s}$ sector, the upgraded experiment aims at a high precision measurement of the $B_{s}$ oscillation phase. Second, where CP violation is being measured in charmless hadronic two-body scalar $B$-decays, the upgraded detector will allow a theoretically clean analysis of vector-vector final states. Third, the precision of the unitarity triangle angle $\gamma$ measurements using tree-level diagrams will be augmented from a value of $4^{\circ}$ with the current $\mathrm{LHCb}$ to $\leq 1^{\circ}$ precision for the upgrade. Fourth, where $\mathrm{CP}$ violation in mixing $\left(A_{f_{s}}\left(B_{s}\right)-A_{f s}\left(B^{0}\right)\right)$ can be studied in LHCb using double semileptonic decays, the upgrade can measure $A_{f s}\left(B_{s}\right)$ and $A_{f s}\left(B^{0}\right)$ individually using full hadronic decays with symmetric charged final states $\left(K^{+} K^{-} \pi^{+} \pi^{-}\right)$, suppressing detector asymmetries.

For rare decay studies, the branching ratios of $B_{s} / B^{0} \rightarrow \mu^{+} \mu^{-}$as well the angular distribution of the final state particles in $B \rightarrow K^{*} \mu^{+} \mu^{-}$offer promising discovery potential for New Physics

Table 1: Sensitivities of the LHCb upgrade to key observables. For each observable the current sensitivity is compared to that expected after LHCb has accumulated $5 \mathrm{fb}^{-1}$ and that which will be achieved with 50 $\mathrm{fb}^{-1}$ by the upgraded experiment, all assuming $\sqrt{s}=14 \mathrm{TeV}$. Note that $S$ represents the time dependent $\mathrm{CP}$-violation amplitude, $s_{0} A_{\mathrm{FB}}$ the zero crossing point of the forward-backward asymmetry, and $\mathscr{A}^{\Delta \Gamma_{s}}$ the $B_{s}$ lifetime-dependent CP violation amplitude.

\begin{tabular}{|c|c|c|c|c|c|}
\hline Type & Observable & $\begin{array}{l}\text { Current } \\
\text { precision }\end{array}$ & $\begin{array}{l}\mathrm{LHCb} \\
\left(5 \mathrm{fb}^{-1}\right)\end{array}$ & $\begin{array}{l}\text { Upgrade } \\
\left(50 \mathrm{fb}^{-1}\right)\end{array}$ & $\begin{array}{c}\text { Theory } \\
\text { uncertainty }\end{array}$ \\
\hline $\begin{array}{l}\text { Gluonic } \\
\text { penguin }\end{array}$ & $\begin{array}{c}S\left(B_{S} \rightarrow \phi \phi\right) \\
S\left(B_{S} \rightarrow K^{* 0} K^{* 0}\right) \\
S\left(B^{0} \rightarrow \phi K_{S}^{0}\right)\end{array}$ & $\begin{array}{c}- \\
- \\
0.17 \\
\end{array}$ & $\begin{array}{l}0.08 \\
0.07 \\
0.15\end{array}$ & $\begin{array}{l}0.02 \\
0.02 \\
0.03 \\
\end{array}$ & $\begin{array}{c}0.02 \\
<0.02 \\
0.02 \\
\end{array}$ \\
\hline$B_{s}$ mixing & $2 \beta_{s}\left(B_{s} \rightarrow J / \psi \phi\right)$ & 0.35 & 0.019 & 0.006 & $\sim 0.003$ \\
\hline $\begin{array}{c}\text { Right-handed } \\
\text { currents } \\
\end{array}$ & $\begin{array}{c}S\left(B_{s} \rightarrow \phi \gamma\right) \\
\mathscr{A}^{\Delta \Gamma_{s}}\left(B_{s} \rightarrow \phi \gamma\right)\end{array}$ & - & $\begin{array}{l}0.07 \\
0.14 \\
\end{array}$ & $\begin{array}{l}0.02 \\
0.03 \\
\end{array}$ & $\begin{array}{c}<0.01 \\
0.02 \\
\end{array}$ \\
\hline $\begin{array}{c}\mathrm{E} / \mathrm{W} \\
\text { penguin }\end{array}$ & $\begin{array}{c}A_{T}^{(2)}\left(B^{0} \rightarrow K^{* 0} \mu^{+} \mu^{-}\right) \\
s_{0} A_{\mathrm{FB}}\left(B^{0} \rightarrow K^{* 0} \mu^{+} \mu^{-}\right)\end{array}$ & - & $\begin{array}{c}0.14 \\
4 \%\end{array}$ & $\begin{array}{c}0.04 \\
1 \%\end{array}$ & $\begin{array}{c}0.05 \\
7 \%\end{array}$ \\
\hline $\begin{array}{l}\text { Higgs } \\
\text { penguin }\end{array}$ & $\begin{array}{c}\mathscr{B}\left(B_{s} \rightarrow \mu^{+} \mu^{-}\right) \\
\frac{\mathscr{B}\left(B^{0} \rightarrow \mu^{+} \mu^{-}\right)}{\mathscr{B}\left(B_{s} \rightarrow \mu^{+} \mu^{-}\right)}\end{array}$ & - & $\begin{array}{c}30 \% \\
-\end{array}$ & $\begin{aligned} & 8 \% \\
& \sim 35 \%\end{aligned}$ & $\begin{array}{l}<10 \% \\
\sim 5 \%\end{array}$ \\
\hline $\begin{array}{l}\text { Unitarity } \\
\text { triangle } \\
\text { angles }\end{array}$ & $\begin{array}{c}\gamma\left(B \rightarrow D^{(*)} K^{(*)}\right) \\
\gamma\left(B_{s} \rightarrow D_{s} K\right) \\
\beta\left(B^{0} \rightarrow J / \psi K^{0}\right)\end{array}$ & $\begin{array}{c}\sim 20^{\circ} \\
- \\
1^{\circ}\end{array}$ & $\begin{array}{l}\sim 4^{\circ} \\
\sim 7^{\circ} \\
0.5^{\circ}\end{array}$ & $\begin{array}{l}0.9^{\circ} \\
1.5^{\circ} \\
0.2^{\circ}\end{array}$ & $\begin{array}{l}\text { negligible } \\
\text { negligible } \\
\text { negligible }\end{array}$ \\
\hline $\begin{array}{l}\text { Charm } \\
\text { CPV }\end{array}$ & $\begin{array}{c}A_{\Gamma} \\
A_{C P}^{d i r}(K K)-A_{C P}^{d i r}(\pi \pi)\end{array}$ & $\begin{array}{l}2.5 \times 10^{-3} \\
4.3 \times 10^{-3} \\
\end{array}$ & $\begin{array}{l}2 \times 10^{-4} \\
4 \times 10^{-4}\end{array}$ & $\begin{array}{l}4 \times 10^{-5} \\
8 \times 10^{-5}\end{array}$ & - \\
\hline
\end{tabular}



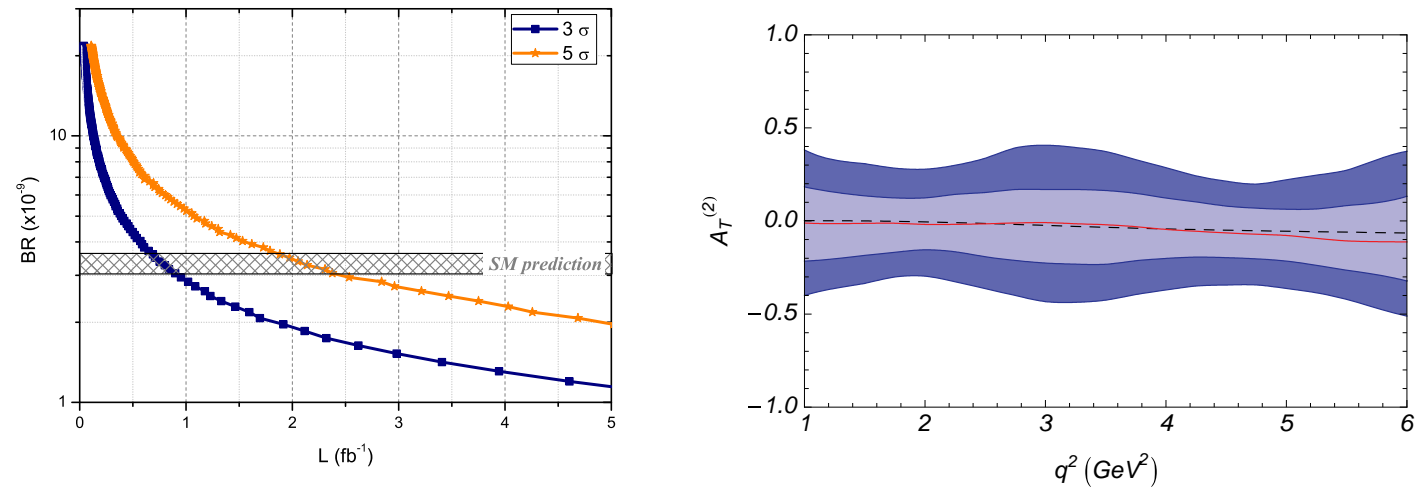

Figure 4: Left: Discovery potential of LHCb in $B_{s} \rightarrow \mu^{+} \mu^{-}$compared to SM predictions as a function of the integrated luminosity at $14 \mathrm{TeV}$ collisions. Right: Expected experimental sensitivity of the transverse asymmetry $A_{T}^{(2)}$ in $B \rightarrow K^{*} \mu \mu$ decays as function of $q^{2}$ of the muon pair, corresponding to $10 \mathrm{fb}^{-1}$ of data.

(NP). Where observation of an enhanced branching ratio of $B_{s} \rightarrow \mu^{+} \mu^{-}$indicates the presence of NP, a comparison to the branching ratio of its sister channel $B^{0} \rightarrow \mu^{+} \mu^{-}$, accessible only with the LHCb upgrade, can distinguish between different NP models [5]. The decay $B \rightarrow K^{*} \mu^{+} \mu^{-}$has a rich phenomenology that includes, in addition to the forward-backward asymmetry observable $A_{F B}$, the transversity asymmetry $A_{T}^{(2)}$ that allows to distinguish between NP models [6]. Fig. 4 (Left) shows the discovery potential of LHCb with $B_{S} \rightarrow \mu^{+} \mu^{-}$and (Right) the expected sensitivity for the $A_{T}^{(2)}$ observable for $10 \mathrm{fb}^{-1}$.

\subsection{Lepton Flavour Physics}

The upgraded LHCb detector can make exciting contributions to the search for flavour violating physics in the lepton sector, in particular searches for heavy Majorana neutrinos and lepton flavour violating $\tau^{ \pm}$decays, forbidden in the SM.

The decay of heavy flavours is a promising approach to search for Majorana neutrinos with a mass in the region of $\approx 1 \mathrm{GeV} / \mathrm{c}^{2}$ [7]. In LHCb two search strategies are foreseen: a direct search for long lived neutrinos produced in $D$ and $B$ decays, and an indirect search looking for same sign charged leptons in $D, B$ and $\tau$ decays with a signature similar to neutrinoless double beta decays. In the first case the produced neutrinos could be detected by a dedicated trigger algorithm if they decay within a distance of $0.5 \mathrm{~m}$, i.e. within the fiducial volume of the Velo. In the second case, relevant $B$-branching ratios are at the level of $10^{-8}-10^{-9}$, which are accessible for LHCb.

The LHCb upgrade will search for lepton flavour violating decays by focussing on charged particle final states, in particular $\tau^{ \pm} \rightarrow \mu^{ \pm} \mu^{+} \mu^{-}$. Current limits for this decay are set to $2.1 \times 10^{-8}$ by the $B$-factories [8], while the LHCb upgrade is expected to improve the sensitivity to the order of $10^{-9}$.

\subsection{Physics Beyond Flavour}

Due to the forward acceptance of the LHCb detector, the upgraded experiment can make important contributions to non-flavour physics, eg. electroweak physics, exotic searches and QCD.

In electroweak physics $\mathrm{LHCb}$ can contribute to the measurement of the electroweak mixing angle for leptons $\sin ^{2} \theta_{e f f}^{\text {lept }}$ and to the measurement of the parton density distributions (PDF) in $W$ boson production. The value of $\sin ^{2} \theta_{\text {eff }}^{\text {lept }}$ can be measured from the forward-backward asymmetry $A_{F B}$ of the leptons produced in $Z$ decays, the magnitude of which is about 5 times higher in the 

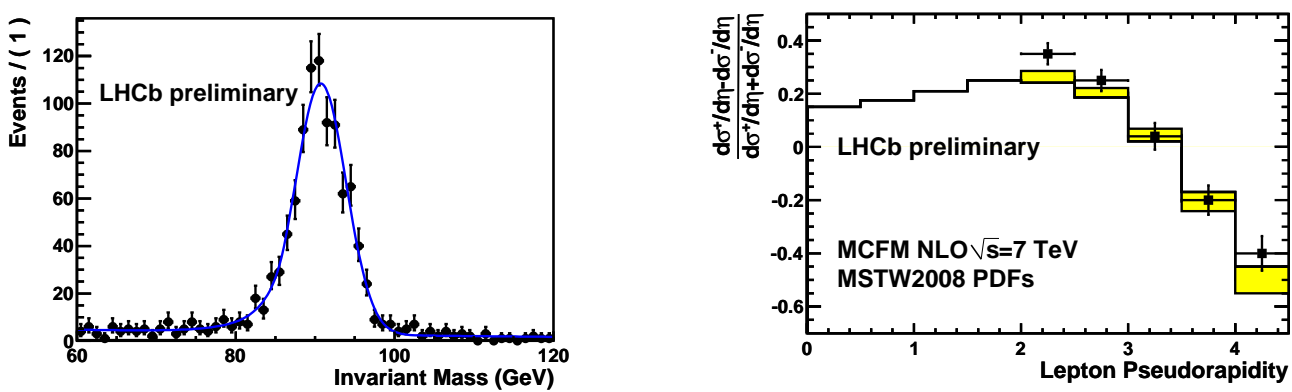

Figure 5: Preliminary $\mathrm{Z}$ and $\mathrm{W}$ results for $16 \mathrm{pb}^{-1}$ of data at $\sqrt{s}=7 \mathrm{TeV}$. Left: the $Z \rightarrow \mu^{+} \mu^{-}$mass peak. Right: the $W^{+}-W^{-}$production asymmetry, where the points are data and the line is the theoretical prediction.

LHC than in LEP. The forward geometry of LHCb is particularly attractive for this measurement since in forward production (rapidity $\eta>3$ ) of a $Z$ from quark - antiquark annihilation, the $Z$ is produced in the direction correlated with the quark direction about $95 \%$ of the time. Preliminary results on $\mathrm{Z}$ and $\mathrm{W}$ physics are illustrated in Fig. 5.

Many NP models predict new particles at the TeV mass scale. LHCb is particularly suited for searches of new, exotic, particles with long lifetimes that are often predicted in these models, since a dedicated trigger line can search for displaced vertices.

In the LHC, an object $X$ can be produced via central exclusive production: $p p \rightarrow p+X+p$, where the + signs indicate a large rapidity gap. Such interactions allow a study of high energy $t$-channel QCD production and low background reconstruction of object $X$, since no other particletracks emerge from the production vertex. The low $p_{T}$ acceptance and high bandwidth of the trigger will allow a wide range of mass states to be collected and analysed in the upgrade.

\section{Conclusions}

The LHCb upgrade will take the next step in sensitivity of flavour physics with $B$ and $D$ mesons. The performance of the current detector demonstrates that a pure event selection is possible at the LHC, even in the presence of pileup. In addition the upgraded detector, with its enhanced trigger and readout scheme, offers exciting possibilities on topics beyond quark flavour physics.

\section{References}

[1] R. Aaij et al., (LHCb Collab.), "The LHCb Upgrade Letter of Intent", CERN/LHCC 2011-001.

[2] A. Augusto Alves Jr et al., (LHCb Collab.), “The LHCb Detector at the LHC”, 2008 JINST 3 S08005.

[3] B. Aubert et al., (BABAR Collab.), Nucl. Intrum. and Meth. A 479 (2002) 1.

[4] M. Anelli et al., "High Radiation tests of the MWPCs for the LHCb muon system", Nucl. Instrum. and Meth. A 599 (2009) 171.

[5] T. Hurth, G. Isidori, J.F. Kamenik and F. Mescia, Nucl. Phys. B 808 (2009) 326; W. Altmannshofer, A.J. Buras, S. Gori, P. Paradisi and D.M. Straub, Nucl. Phys. B 830 (2010) 17.

[6] U. Egede, T. Hurth, J. Matias, M. Ramon and W. Reece, JHEP 0811 (2008) 032.

[7] A. Atre, T. Han, S. Pascoli and B. Zhang, JHEP, 0905:030 (2009); A. Atre, V. Bargerand and T. Han, Phys. Rev. D71, 113014 (2005); D. Gorbunov and M. Shaposhnikov, JHEP 0710 (2007) 0.15.

[8] B. Aubert et al., (BABAR Collab.), Phys. Rev. Lett. 104 (2010) 021802; K. Hayasakai et al., (Belle Collaboration), Phys. Lett. B 687 (2010) 139. 